\title{
A Specific Synaptic Pathway Activates a Conditional Plateau Potential Underlying Protraction Phase in the Aplysia Feeding Central Pattern Generator
}

\author{
Nikolai C. Dembrow, Jian Jing, Vladimir Brezina, and Klaudiusz R. Weiss \\ Department of Physiology and Biophysics, Mount Sinai School of Medicine, New York, New York 10029
}

\begin{abstract}
A common feature in the architecture of neuronal networks is a high degree of seemingly redundant synaptic connectivity. In many cases, the synaptic inputs converging on any particular neuron all use the same neurotransmitter and appear to be fundamentally equivalent. Here, we analyze a striking counterexample in which such inputs are not equivalent and, as a result, play very different roles in the generation of the pattern of activity produced by the network. In the feeding central pattern generator of Aplysia, the pattern-initiating neuron B50 elicits motor programs by exciting the plateauing neuron B31/B32 in two ways: directly and indirectly through neuron B63. All of the synaptic connections use ACh. Despite the direct input of B50 to B31/B32, the indirect pathway of exciting B31/B32 through B63 is required for $\mathrm{B} 50$ to elicit the $\mathrm{B} 31 / \mathrm{B} 32$ plateau potential and the motor program. We dissect this requirement using the muscarinic cholinergic antagonist pirenzepine. Pirenzepine blocks the B50-elicited motor program, the plateau potential in B31/B32, and, notably, a slow component of the EPSP elicited in B31/B32 by B63 but not that elicited by B50. The muscarinic agonist oxotremorine restores the plateau potential in $\mathrm{B} 31 / \mathrm{B} 32$ and eliminates the necessity for $\mathrm{B} 63$ in $\mathrm{B} 50$-elicited motor programs. Together, our analysis shows that the plateau potential in B31/B32 is not endogenous but conditional, furthermore conditional on one particular synaptic input, that from B63. Thus, among several inputs to B31/B32 that use the same transmitter, the input from B63 is functionally distinct in its preferential access to the plateau potential that represents the committed step toward the initiation of a motor program.
\end{abstract}

Key words: conditional plateau potential; neuronal networks; Aplysia; feeding circuitry; convergence; muscarinic action

\section{Introduction}

Many studies seek to understand how the architecture of neuronal networks relates to their ability to produce coherent patterns of activity. Commonly, these networks possess a high degree of seemingly redundant synaptic connectivity. Information from one neuron can typically reach another neuron through several synaptic pathways. In many cases, the synaptic connections involved all use the same neurotransmitter. The synaptic inputs converging on the target neuron all appear to be fundamentally equivalent, with synaptic strength being the only major distinguishing factor. This picture is the basis of many conceptualizations of how such networks function (Amit, 1989; Kristan and Shaw, 1997; Abbott and Sejnowski, 1999). However, this picture may not always be correct. We describe here a case in which two synaptic inputs that use the same transmitter and appear equivalent in fact exert qualitatively different actions on the target neuron. As a result, information from an upstream neuron propagating through the two pathways has very different functional significance.

Received Dec. 22, 2003; revised April 30, 2004; accepted April 30, 2004

This work was supported by National Institute of Mental Health Grants MH50235 and K05 MH01427 to K.R.W. and National Institute of Neurological Disorders and Stroke Grant NS41497 to V.B.

Correspondence should be addressed to Dr. Klaudiusz Weiss, Department of Physiology and Biophysics, Box 1218, Mount Sinai School of Medicine, New York, NY 10029. E-mail: klaudiusz.weiss@mssm.edu.

DOI:10.1523/JNEUROSCI.5649-03.2004

Copyright $\odot 2004$ Society for Neuroscience $\quad$ 0270-6474/04/245230-09\$15.00/0
In the feeding central pattern generator (CPG) of Aplysia, neurons that initiate patterned activity do so by activating a plateau potential in buccal neuron B31/B32. The induction of this plateau potential has been described as the neural event underlying the committed decision to perform a motor program (Susswein et al., 2002). Pattern-initiating neurons such as the cerebralto-buccal interneuron 2 (CBI-2) and the buccal interneuron B50 excite B31/B32 through two seemingly redundant pathways: directly and indirectly through buccal interneuron B63 (Hurwitz et al., 1997, 2003; Dembrow et al., 2003). Inputs to B31/B32 from all three neurons, $\mathrm{CBI}-2, \mathrm{~B} 50$, and B63, appear to be cholinergic (Dembrow et al., 2003; Hurwitz et al., 2003). Each of the three neurons elicits both fast and slow EPSPs in B31/B32. It might, therefore, be thought that the direct and indirect pathways should both be equally capable of inducing the plateau potential in B31/B32. Previously, it was found that CBI-2 requires B63 activity to elicit motor programs, but this could be attributable to the fact that the CBI-2-elicited EPSPs in B31/B32 are smaller than the B63-elicited EPSPs (Hurwitz et al., 2003). Here, we worked with B50, the EPSPs of which are of the same magnitude as those of B63 (Dembrow et al., 2003; Hurwitz et al., 2003). We found that $\mathrm{B} 50$, too, requires $\mathrm{B} 63$ activity to induce plateau potentials in $\mathrm{B} 31 / \mathrm{B} 32$ and elicit motor programs. We show that this is because the plateau potential in B31/B32 is not an endogenous but rather a conditional plateau, furthermore selectively conditional on just one of the inputs to B31/B32, that from B63. Thus, by having 
privileged access to the plateau potential, the indirect pathway through $\mathrm{B} 63$ has a specific functional significance in the initiation of motor programs.

Some of these results have been presented previously in an abstract (Dembrow et al., 2002).

\section{Materials and Methods}

General. Experiments were performed on Aplysia californica weighing 50-250 gm, obtained from Marinus (Long Beach, CA). Animals were maintained for $2-7 \mathrm{~d}$ in holding tanks at $14-16^{\circ} \mathrm{C}$ until use. Before dissection, animals were anesthetized by injection of $50 \%$ of their body weight of isotonic $\mathrm{MgCl}_{2}$ into the body cavity. Buccal ganglia were removed from the animal and desheathed in 50\% artificial seawater (ASW) and $50 \%$ isotonic $\mathrm{MgCl}_{2}$. For recording, the ganglia were pinned caudal surface up in a dish containing 100\% ASW (in mM: $460 \mathrm{NaCl}, 10 \mathrm{KCl}, 11$ $\mathrm{CaCl}_{2}, 55 \mathrm{MgCl}_{2}$, and $20 \mathrm{HEPES}$, at pH 7.5). Fresh ASW was perfused into the dish at a rate of $375 \mu \mathrm{l}$ ( $25 \%$ of dish volume)/min with a peristaltic pump (Dynamax; Rainin, Ridgefield, NJ) and removed using vacuum suction. The dish was cooled to $14-16^{\circ} \mathrm{C}$.

Hexamethonium, oxotremorine (OXO), pirenzepine (PIR), and TTX were applied by adding them to the perfusate. To reduce polysynaptic input to B31/B32, a high divalent cation solution (HiDi; in mм: 312 $\mathrm{NaCl}, 10 \mathrm{KCl}, 33 \mathrm{CaCl}_{2}, 132 \mathrm{MgCl}_{2}$, and 20 HEPES, at $\mathrm{pH} \mathrm{7.5)} \mathrm{was}$ perfused instead of ASW. In reduced sodium solutions, sodium was substituted with $\mathrm{N}$-methyl-D-glucamine (NMDG). All chemicals were obtained from Sigma (St. Louis, MO).

Electrophysiological recordings. Intracellular recordings were made using single-barreled glass microelectrodes filled with $2 \mathrm{~m} \mathrm{~K}$-acetate and 30 $\mathrm{mm} \mathrm{KCl}$, beveled to a resistance of 5-8 $\mathrm{M} \Omega$. Recordings were obtained using either an Axoclamp 2A or 2B (Axon Instruments, Foster City, CA) or a Getting 5A amplifier (Getting Instruments, Iowa City, IA). Neurons were stimulated with repeated short pulses using a model S88 stimulator (Grass Medical Instruments, Quincy, MA), whereas the Getting or Axoclamp amplifiers were used to perform longer DC injections.

Extracellular nerve recordings were obtained using polyethylene suction electrodes connected to a differential AC amplifier (M1700; A-M Systems, Everett, WA). The Grass S88 stimulator was used for nerve stimulation.

All electrophysiological data were recorded on a chart recorder (MT9500; Astro-Med, West Warwick, RI) as well as a chart modulator recorder (VDAT8; Vetron Technology, Howard, PA). Data were digitized using a DigiData 1322A with AxoScope software (Axon Instruments). Digitized data were analyzed using ClampFit 8.1 (Axon Instruments) and compiled into figures using CorelDraw (Corel, Dallas, TX).

Voltage-clamp experiments. To record B50-elicited synaptic currents, B31/B32 neurons were voltage clamped using the single-electrode voltage clamp (SEVC) mode of the Axoclamp amplifier. B31/B32 was held at either -80 or $-40 \mathrm{mV}$ while B50 was stimulated with $10 \mathrm{msec}$ suprathreshold depolarizing pulses at $15 \mathrm{~Hz}$ for $1 \mathrm{sec}$. These experiments were performed in HiDi solution to minimize polysynaptic activity.

Voltage-ramp experiments. To characterize currents underlying the B31/B32 plateau potential, we performed a series of voltage-ramp experiments. Each hemiganglion contained a B31 and a B32 neuron. All four B31/B32 neurons in the ganglion were electrically coupled. To minimize the effects of this coupling, we used two strategies. First, the ganglion was split at the commissure to remove the contralateral B31/32 neurons. Second, two SEVC amplifiers were used to simultaneously clamp both of the remaining B31/B32 neurons. Each neuron was given identical voltage-ramp commands, thereby minimizing the instantaneous voltage differential and hence current spread between the neurons. Clearly, complete isolation of the two neurons by this technique would only have been possible in the ideal situation of completely symmetrical neuronal morphologies and voltage-clamp gain. Nevertheless, the technique mostly succeeded in eliminating the uncontrolled surges of current that were visible when the technique was not used. TTX was perfused into the bath to minimize action potential-mediated synaptic input. To determine the quasi-steady-state current-voltage ( $I-V)$ relationship, we used slowly depolarizing voltage ramps from -80 to $-20 \mathrm{mV}$ at $0.5 \mathrm{mV} / \mathrm{sec}$.
Measurements and statistics. For the purpose of measuring its duration, the plateau potential in B31/B32 was taken to end when the membrane potential passed again the value of the resting potential before the plateau potential was elicited. This provided an unambiguous end point because, in almost all cases, the depolarized plateau potential was immediately succeeded by a well defined afterhyperpolarization.

For all experiments, the sample size, $n$, is the number of preparations. All group data are reported as mean $\pm \mathrm{SE}$, with the sample size, $n$, being the number of individual observations, in some cases from a smaller number of preparations.

\section{Results}

The consummatory feeding behaviors of Aplysia consist of the cyclical repetition of several sets of movements by a hand-like organ called the radula. Within each cycle of movement, the radula first protracts outward and then retracts back into the mouth (Kupfermann, 1974). These movements are coordinated by cycles of patterned activity, or motor programs, generated by the feeding CPG. In the isolated feeding circuitry, motor programs can be monitored by the activity of several specific motor neurons and/or the peripheral nerves that contain their processes. Radula protraction is monitored by activity in the I 2 nerve (I2N) and radula retraction by activity in buccal nerve 2 (BN2) (Morton and Chiel, 1993; Hurwitz et al., 1994). Protraction and retraction monitored in this way are marked by the white and black bars, respectively, below the traces in Figure 1 and subsequent figures. The neurons that we focus on here, CBI-2, B50, $\mathrm{B} 63$, and $\mathrm{B} 31 / \mathrm{B} 32$, constitute the core of the circuit that initiates and maintains protraction.

\section{$\mathrm{B} 50$ requires $\mathrm{B} 63$ firing to drive motor programs}

Previous work has shown that the pattern-initiating neuron CBI-2 fails to elicit motor programs when B63 firing is blocked (Hurwitz et al., 1997, 2003). We sought to determine whether the other pattern-initiating neuron, B50, also required B63 firing to drive motor programs. Indeed, this was the case (Fig. 1). Stimulating B50 for the duration of the protraction phase normally resulted in a single-cycle motor program (Fig. $1 \mathrm{~A}$, left). Hyperpolarization of a single B63 (gray bar) before and during the B50 stimulation prevented the motor program, although B50 was stimulated for a longer period (Fig. $1 A$, middle) $(n=4)$. The peak depolarization in B31/B32 during the B50 stimulation was significantly less when B63 was hyperpolarized (27.3 \pm 2.5 vs $51.1 \pm 1.4 \mathrm{mV}$ under control conditions; B31/B32 depolarization: ANOVA, $F_{(4,24)}=30.27, p<0.0001$; Bonferroni's multiple comparison tests, $p<0.001$ ), and no plateau potential appeared to occur (see below). Protraction-phase nerve activity was reduced (I2N) and retraction-phase nerve activity was entirely blocked (BN2). The same result was obtained when B50 was stimulated continuously to drive several program cycles (Fig. $1 B$ ) $(n=3)$. Thus, although B50 elicits direct EPSPs in B31/B32 that are even larger than those elicited by B63 (Dembrow et al., 2003; Hurwitz et al., 2003), selective elimination of the indirect pathway through $\mathrm{B} 63$ blocks the ability of B50 to elicit motor programs.

How we recognized the presence or absence of the plateau potential in B31/B32 in these programs (and similarly in Figs. 3 and 10) requires some comment. The plateau potential is an all-or-none event (Hurwitz et al. 1994; Susswein et al. 2002), and as such it was easily recognized, for example, when triggered by DC injection (see Figs. 4-8). In the case of CBI-2-elicited motor programs, likewise, hyperpolarization of B63 clearly eliminated the plateau potential in an all-or-none manner (Hurwitz et al. 1997). With the B50-elicited programs, however, the elimination 

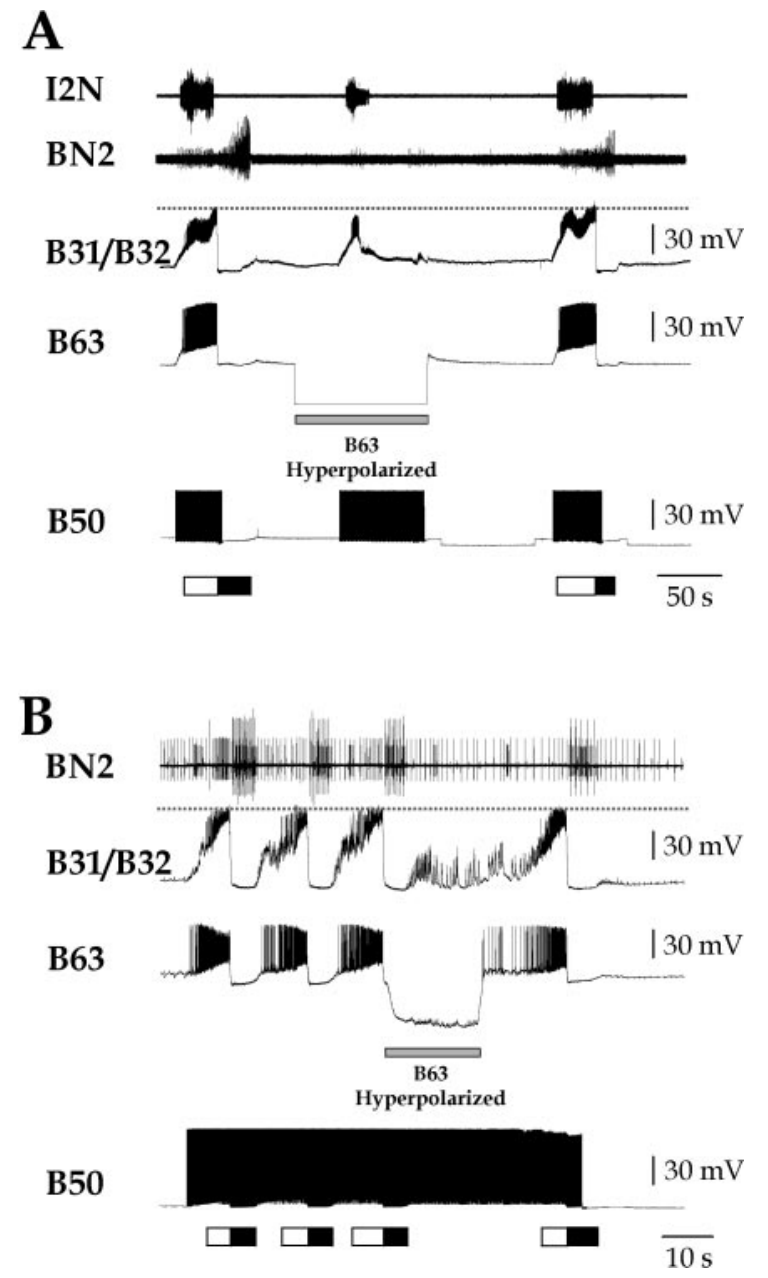

Figure 1. $B 50$ requires $B 63$ firing to drive motor programs. Single $(A)$ or multicycle $(B)$ programs were elicited by stimulating $B 50$ with brief current pulses at $10 \mathrm{~Hz}$. The protraction phase (marked by white bar below traces) was monitored by intracellular recording of B31/B32 and extracellular recording of the 12 nerve (I2N). The retraction phase (marked by black bar below traces) was monitored by extracellular recording of the buccal nerve 2 (BN2). When B63 was hyperpolarized (gray bar), B31/B32 no longer entered plateau, I2N activity was severely reduced, and BN2 activity was completely blocked. The dashed line marks the level of peak depolarization in B31/B32 under control conditions to facilitate comparison.

of the plateau potential was obscured by the fact that B31/B32 still continued to be depolarized by summating fast EPSPs. But the depolarization was always significantly smaller when the plateau potential was eliminated and, furthermore, never exhibited the characteristic abrupt secondary rise to the plateau level (see Fig. 10, OXO) (Hurwitz et al., 1997, 2003; Susswein et al., 2002) but instead often declined (Figs. 1, 3). Finally, the activity in the I2N nerve, which carries axons of B31/B32 and so reflects the prolonged train of spikes that normally accompanies the B31/B32 plateau potential (Hurwitz et al., 1994, 1996), was usually eliminated in an all-or-none manner (see Figs. 3, 10).

\section{B50-elicited slow EPSP in B31/B32 is not PIR sensitive}

The neurons B50, CBI-2, and B63 all appear to use ACh, because each neuron elicits fast EPSPs in B31/B32 that are blocked by the specific nicotinic antagonist hexamethonium (Dembrow et al., 2003; Hurwitz et al., 2003). In addition to these fast EPSPs, each neuron also elicits a slow, hexamethonium-insensitive EPSP in $\mathrm{B} 31 / \mathrm{B} 32$. In the case of B63, this slow EPSP is blocked by the muscarinic antagonist PIR (I. Hurwitz, R. A. DiCaprio, and K. R.
A

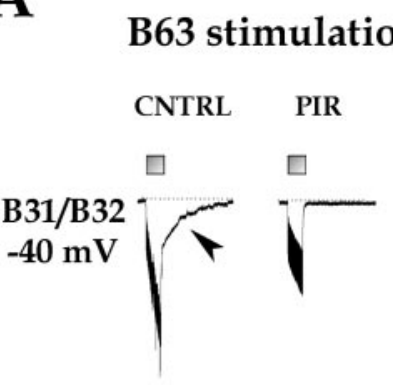

\section{B50 stimulation}
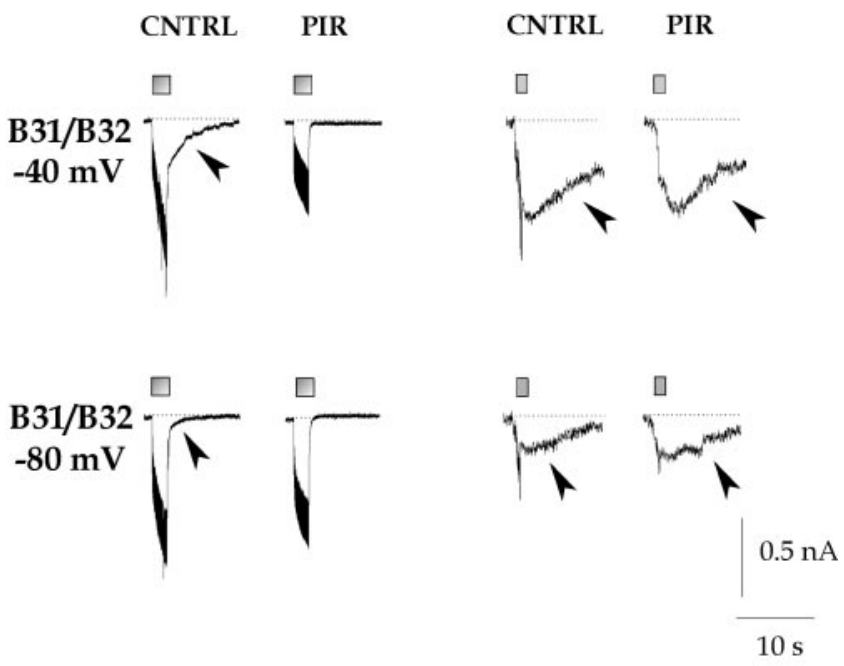

B

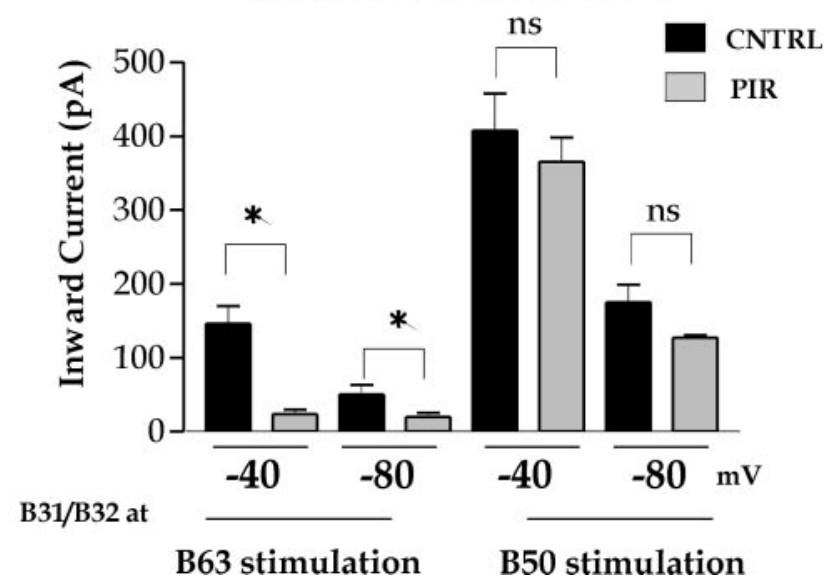

Figure 2. B50-elicited slow EPSP in B31/B32 is not PIR sensitive. A, Representative records. $B 63$ (left) or $B 50$ (right) was stimulated with short trains of high-frequency depolarizing pulses (B63: $2 \mathrm{sec}, 20 \mathrm{~Hz}$; B50: $1 \mathrm{sec}, 20 \mathrm{~Hz}$; both marked by gray rectangles), whereas B31/B32 was voltage clamped at -80 or $-40 \mathrm{mV}$, in the presence of only $10^{-5} \mathrm{M}$ hexamethonium (CNTRL) or $10^{-5} \mathrm{~m}$ hexamethonium and $10^{-3} \mathrm{~m}$ pirenzepine (PIR). The arrowheads indicate the slow component of synaptic input. $B$, Group data of inward current measured $500 \mathrm{msec}$ after the end of the synaptic stimulation from records like those in $A$. The asterisks indicate statistically significant differences; $n$ in indicates no significant difference (see Results). For the eight bars from left to right, $n=28,46,23,27,18,22,9$, and 8 , respectively.

Weiss, unpublished observations). In view of the apparent difference in functional efficacy of the two synaptic inputs, we sought to determine whether the B50-elicited slow EPSP in B31/B32 was PIR sensitive as well.

We examined the synaptic currents in B31/B32 elicited by B50 stimulation while B31/B32 was voltage clamped at different holding potentials in the absence and presence of PIR. For comparison, we examined the currents elicited by stimulation of B63. In both cases, hexamethonium was present to block the fast component and thereby emphasize the slow component of both synaptic inputs. Figure $2 A$ shows typical slow synaptic currents elicited by B63 and B50 (Fig. 2A, arrowheads). For quantitative comparison, we measured the inward current that persisted $500 \mathrm{msec}$ after the termination of the synaptic stimulation. Figure $2 B$ 


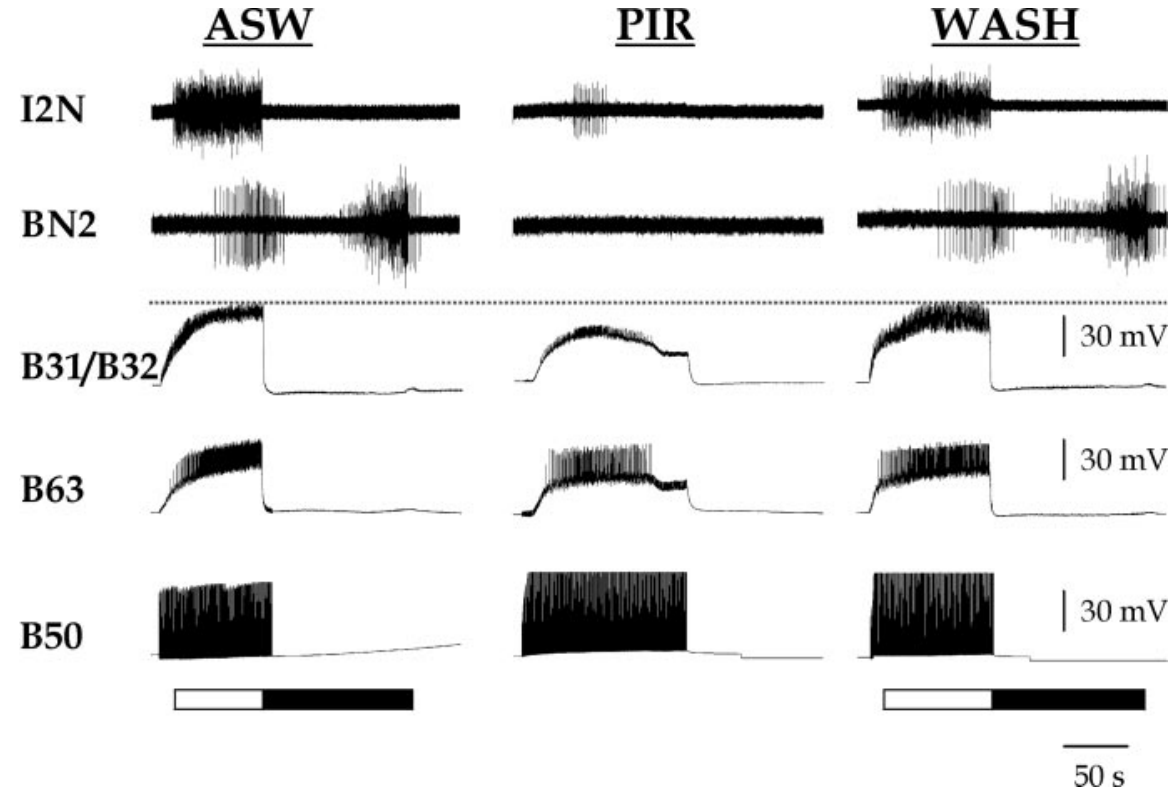

Figure 3. B50-elicited motor programs are blocked by PIR. Single-cycle programs were triggered once every minute by stimulating B50 at $10 \mathrm{~Hz}$, in normal saline (ASW), in $1 \times 10^{-3} \mathrm{M}$ pirenzepine (PIR), and after normal saline wash (WASH). The protraction phase (marked by white bar below traces) was monitored by firing of neurons B31/B32 and B63 and activity in the $\mathrm{I} 2$ nerve (I2N). The retraction phase (marked by black bar below traces) was monitored by activity in the buccal nerve 2 (BN2) and hyperpolarization in B63 and B31/B32. The dashed line marks the level of peak depolarization in B31/B32 under control conditions to facilitate comparison.

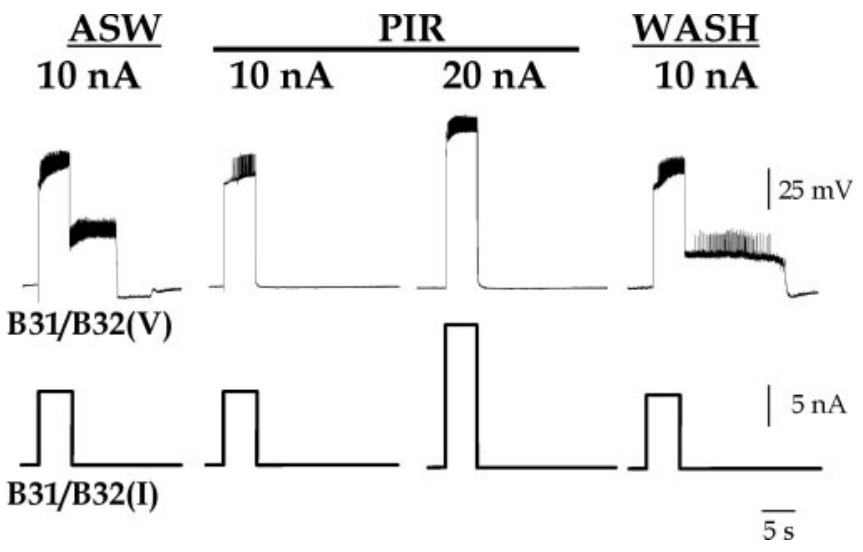

Figure 4. Plateau potential induced in B31/B32 by DC injection is blocked by PIR. Depolarizing current of 10 or $20 \mathrm{nA}$ was injected for 5 sec into B31/B32 (I) and its voltage response $(V)$ was recorded in normal saline (ASW), in $1 \times 10^{-3} \mathrm{M}$ pirenzepine (PIR), and after normal saline wash (WASH). In PIR, there was no plateau potential in B31/B32 even when twice the amount of current sufficient to elicit a plateau potential in normal saline was injected.

shows the group data from a number of experiments (sample sizes are listed in the legend to Fig. 2). For both inputs, the slow component was significantly larger when B31/B32 was held at $-40 \mathrm{mV}$ than when it was held at $-80 \mathrm{mV}\left(\right.$ ANOVA: $F_{(7,173)}=$ 46.67, $p<0.0001$; Bonferroni's multiple comparison test: B63, $p<0.05$; B50, $p<0.001)$. The slow component elicited by B50 was significantly larger than that elicited by B63, whether B31/ B32 was held at $-40 \mathrm{mV}(p<0.001)$ or $-80 \mathrm{mV}(p<0.01)$. Most importantly, the perfusion of PIR $\left(10^{-3} \mathrm{M}\right)$ significantly reduced the B63-elicited slow component at $-40 \mathrm{mV}(p<$ $0.001)$ as well as $-80 \mathrm{mV}(p<0.05)$ (Fig. $2 B$, asterisk), confirming the previous results of Hurwitz, DiCaprio, and Weiss (unpublished observations). In contrast, the B50-elicited slow component was not significantly affected by $10^{-3} \mathrm{M}$ PIR at $-40 \mathrm{mV}$ $(p>0.05)$ or $-80 \mathrm{mV}(p>0.05)$. Thus, the B50-elicited slow EPSP is PIR insensitive and different, in this respect, from the slow EPSP elicited by B63.

\section{B50-elicited motor programs are blocked by PIR}

As described above, we found that B50 required B63 firing to elicit motor programs (Fig. 1). Perhaps this was in some way connected with the fact that the B63-elicited slow EPSP in B31/B32 was PIR sensitive, whereas the direct B50-elicited EPSP was not. To test this, we examined whether PIR blocked the ability of B50 to elicit motor programs.

Indeed, programs elicited by B50 stimulation in normal saline (Fig. 3, ASW) were blocked in the presence of PIR, although B50 was stimulated for a longer period $(n=3)$. Both protraction-phase (I2N) and retraction-phase (BN2) activity was weak. Although B31/B32 continued to be depolarized by fast, short-latency EPSPs, the peak depolarization was significantly smaller than in normal saline $(22.3 \pm 1.3$ vs $51.1 \pm 1.4 \mathrm{mV}$; B31/B32 depolarization ANOVA as above; Bonferroni's multiple comparison test, $p<$ 0.001 ) and no plateau potential appeared to be induced. B63 was also less depolarized in the presence of PIR. This reduced B63 depolarization may have been a consequence of the PIR-induced block of the plateau potential in B31/B32, to which B63 is electrically coupled. Nevertheless, note in Figure 3 that B63 continued to fire robustly even in the presence of PIR (at $12.7 \pm 1.8 \mathrm{~Hz} ; n=$ $3)$. This supported the idea that it was the slow PIR-sensitive EPSP elicited by B63 in B31/B32, rather than any upstream factor, that was the critical PIR-sensitive component.

\section{Plateau potential induced in $\mathrm{B} 31 / \mathrm{B} 32$ by $\mathrm{DC}$ injection is blocked by PIR}

Injecting B31/B32 with depolarizing current of sufficient magnitude and duration induces a plateau potential in normal saline (Susswein and Byrne, 1988; Hurwitz et al., 1994; Susswein et al., 2002). Current injected into B31/B32 transfers via electrical coupling with B63 (Hurwitz et al., 1997), which in turn synaptically excites B31/B32. We sought to determine whether plateau potentials induced in this way were also PIR sensitive.

Indeed, as shown in Figure 4, PIR blocked the ability of DC injection to trigger a plateau potential in B31/B32. In normal saline (ASW), injecting $10 \mathrm{nA}$ of depolarizing current into B31/ B32 triggered a plateau potential that persisted for $18.3 \pm 1.8 \mathrm{sec}$ $(n=3)$ before returning to the resting membrane potential. The perfusion of PIR prevented the plateau from forming at all in response to the $10 \mathrm{nA}$ and even $20 \mathrm{nA}$ current injections. The block reversed with the removal of PIR (Fig. 4, WASH); the plateau potential returned with durations even longer than under control conditions $(44.2 \pm 4.2 \mathrm{sec})$.

Plateau potential induction in a pharmacologically isolated $\mathrm{B} 31 / \mathrm{B} 32$ is restored by $\mathrm{OXO}$

Minimizing synaptic input to B31/B32 with TTX also prevents DC injections into B31/B32 from triggering plateau potentials 


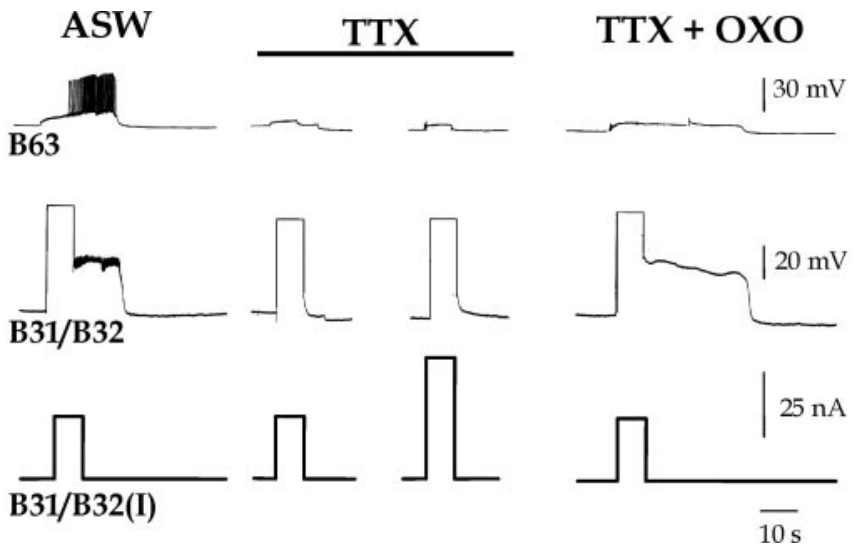

Figure 5. Oxotremorine $(0 X 0)$ restores the ability of $D C$ injection to induce a plateau potential in B31/B32 in the presence of tetrodotoxin (TTX). A depolarizing current of 25 or $50 \mathrm{nA}$ was injected for 10 sec into $B 31 / B 32$, while recording from the contralateral, electrically coupled $B 63$ in normal saline (ASW), $3.3 \times 10^{-5} \mathrm{MTX}$, and $3.3 \times 10^{-5} \mathrm{M} \mathrm{TTX}$ with $10^{-5} \mathrm{M}$ OXO (TTX + OXO).

(Hurwitz, DiCaprio, and Weiss, unpublished observations). In B31/B32 that was pharmacologically isolated from synaptic input in this way, we examined whether the plateau potential induction could be restored by the muscarinic agonist OXO.

DC injections into B31/B32 recruited firing of B63 and induced the plateau potential in B31/B32 (Fig. 5, ASW). When the plateau potential terminated, a subsequent retraction phase occurred (indirectly monitored by hyperpolarization in B31/B32 and B63). TTX effectively blocked the B63 firing and the plateau potential induction in B31/B32 (Fig. 5, TTX) $(n=15)$, even when B31/B32 was injected with current double that which had been sufficient to induce the plateau potential in ASW $(n=3)$. Neither was there any subsequent retraction phase in the presence of TTX. The subsequent addition of OXO $\left(10^{-5} \mathrm{M}\right)$ then restored the ability of the current injection to induce the B31/B32 plateau potential (Fig. 5, TTX + OXO) $(n=12)$, whereas B63 continued to be silent.

We also examined whether minimizing synaptic input using a high divalent cation solution (HiDi) would block the plateau potential and whether OXO would restore it. Indeed, HiDi also effectively prevented B31/B32 plateau potential induction (Fig. 6) $(n=6)$ and the recruitment of a subsequent retraction phase (monitored in Fig. 6 by the firing of neuron B4/5) in response to B31/B32 current injection. The addition of OXO restored the B31/B32 plateau potential. This was the case whether HiDi was used alone $(n=6)$ or in combination with TTX $(n=7)$ (Fig. 6).

The plateau potential restored by $\mathrm{OXO}$ was indistinguishable from the plateau potential induced in normal saline. The duration of the plateau potential was similar in normal saline $(19.8 \pm$ $1.5 \mathrm{sec} ; n=17)$ and when the plateau potential was induced by OXO in the presence of TTX (24.1 $\pm 1.5 \mathrm{sec} ; n=19)$, HiDi $(23.4 \pm 1.7 \mathrm{sec} ; n=12)$, or HiDi and TTX $(20.8 \pm 1.2 \mathrm{sec} ; n=$ 22). Likewise, the maximal depolarization during the plateau potential was similar in normal saline $(34.6 \pm 5.3 \mathrm{mV} ; n=11)$ and when the plateau potential was induced by $\mathrm{OXO}$ in the presence of TTX $(36.1 \pm 2.0 \mathrm{mV} ; n=15), \mathrm{HiDi}(36.1 \pm 1.9 \mathrm{mV} ; n=8)$, or HiDi and TTX $(33.7 \pm 5.0 \mathrm{mV} ; n=10)$. In neither parameter did the four conditions differ significantly (plateau duration: ANOVA: $F_{(3,66)}=1.51, p=0.23$; plateau amplitude: $F_{(3,43)}=$ $0.203, p=0.81)$. Importantly, the plateau potential restored by OXO could then be blocked by PIR (Fig. 7) $(n=5)$.

Together, our data so far suggested that the B31/B32 plateau potential is conditional on synaptic input, specifically a
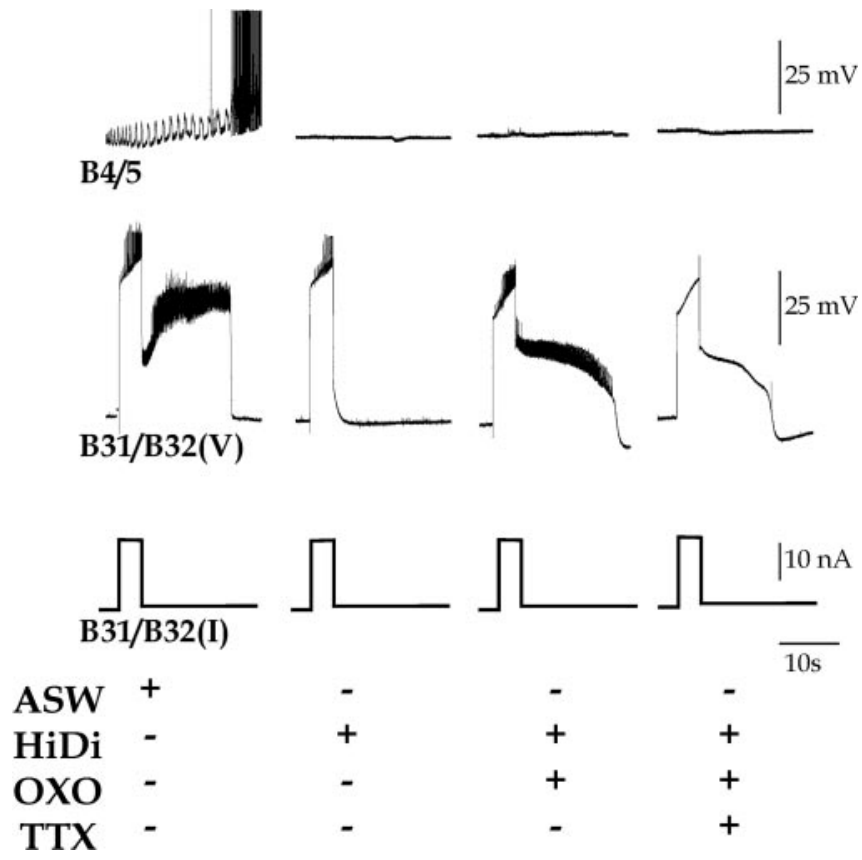

Figure 6. Oxotremorine $(0 X 0)$ restores the ability of $D C$ injection to induce a plateau potential in B31/B32 in the presence of a high divalent cation solution (HiDi) and tetrodotoxin (TTX). A depolarizing current of $20 \mathrm{nA}$ was injected for 5 sec into $\mathrm{B} 31 / \mathrm{B} 32$, while recording from neuron $\mathrm{B} 4 / 5$ to monitor retraction phase, in normal saline (ASW), HiDi, HiDi with $10^{-5} \mathrm{M} 0 \mathrm{XO}$, and HiDi with $3.3 \times 10^{-5} \mathrm{M} \mathrm{TTX}$ and $10^{-5} \mathrm{M}$ OXO.

\section{TTX + OXO $\stackrel{\text { PIR }}{\text { TIR OXO }} \stackrel{\text { PIR WASH }}{\text { TTX + OXO }}$}

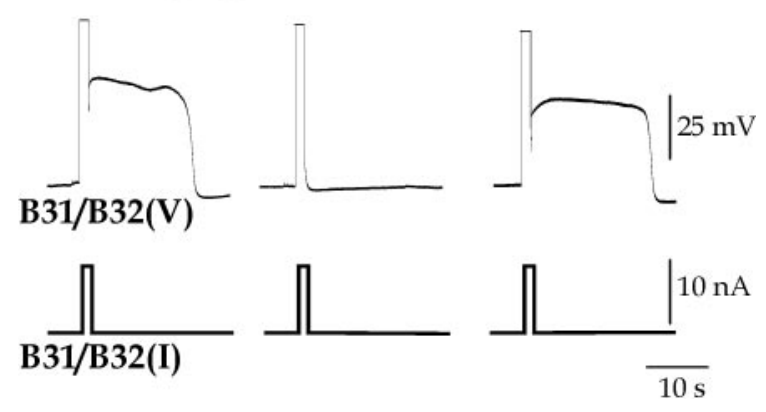

Figure 7. The oxotremorine (OX0)-restored plateau is blocked by pirenzepine (PIR). A depolarizing current of $10 \mathrm{nA}$ was injected for 2 sec into B31/B32 in normal saline with $3.3 \times$ $10^{-5} \mathrm{M}$ tetrodotoxin (TTX) and $10^{-5} \mathrm{MOXO}(\mathrm{TTX}+\mathrm{OXO})$, then with the addition and following wash of $10^{-3} \mathrm{M}$ PIR.

muscarinic-like input, most likely that from B63, that is blocked by PIR and pharmacologically activated by OXO.

\section{The OXO-restored plateau potential is dependent on external sodium}

For a plateau potential to be maintained, a net inward current must be activated. To test whether sodium ions might be necessary for this inward current, we substituted external sodium with NMDG.

Substitution of external sodium with NMDG blocked the OXO-restored plateau potential (Fig. 8). NMDG did not appear to have a direct pharmacological effect on the plateau potential, because even in the presence of $280 \mathrm{~mm} \mathrm{NMDG}\left(50 \% \mathrm{Na}^{+} / 50 \%\right.$ $\mathrm{NMDG})$ the plateau potential persisted for $22.8 \pm 2.6 \mathrm{sec}(n=9)$, with a maximal depolarization of $36.2 \pm 0.3 \mathrm{mV}(n=7)$, values 

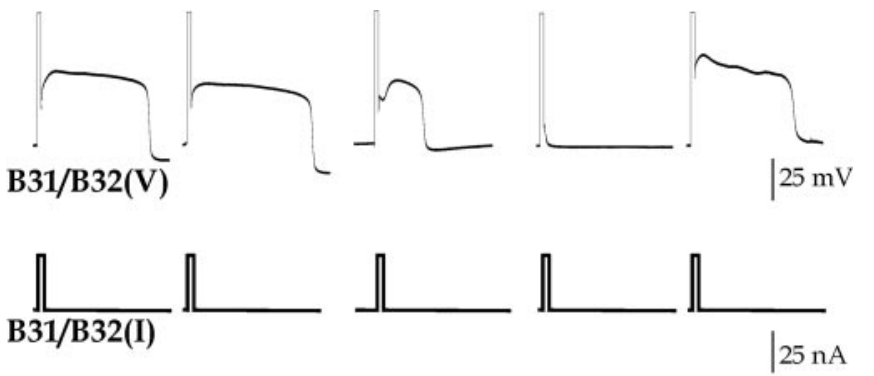

$\begin{array}{cllcc}100 \% \mathrm{Na} & 50 \% \mathrm{Na} & 25 \% \mathrm{Na} & 0 \% \mathrm{Na} & \begin{array}{c}10 \mathrm{~s} \\ 100 \mathrm{Na}\end{array} \\ 0 \% \mathrm{NMDG} & 50 \% \mathrm{NMDG} & 75 \% \text { NMDG } & 100 \% \mathrm{NMDG} & 0 \% \mathrm{NMDG}\end{array}$

\section{TTX + OXO}

Figure 8. The 0X0-restored plateau is dependent on external sodium. A depolarizing current of $20 \mathrm{nA}$ was injected for 3 sec into B31/B32 in normal saline (100\% Na/0\% NMDG) containing $3.3 \times 10^{-5} \mathrm{M} \mathrm{TTX}$ and $10^{-5} \mathrm{M} 0 \mathrm{OX}$, and then while progressively substituting external sodium with NMDG.

not significantly different from those in normal, $100 \% \mathrm{Na}^{+}$saline (above) (plateau duration: ANOVA: $F_{(3,47)}=4.732, p<0.01$; Bonferroni's multiple comparison test, $p>0.05$; plateau amplitude: ANOVA: $F_{(3,39)}=25.87, p<0.0001$; Bonferroni's multiple comparison test, $p>0.05)$. However, in $25 \% \mathrm{Na}^{+} / 75 \% \mathrm{NMDG}$, the plateau potential was significantly reduced in both duration $(11.7 \pm 2.8 \mathrm{sec} ; n=9 ; p<0.01)$ and amplitude $(17.2 \pm 1.9 \mathrm{mV}$; $n=10 ; p<0.001)$. In $0 \% \mathrm{Na} / 100 \% \mathrm{NMDG}$, the plateau potential was completely blocked (Fig. 8). The plateau potential returned when external sodium was restored.

\section{Oxotremorine induces an inward current in B31/B32}

Although some of the properties of the inward current could already be inferred from the characteristics of the membrane potential changes, we sought to measure the inward current directly using voltage clamp. We examined whether the application of OXO induced a component of inward current in the $I-V$ relationship of B31/B32 in the presence of TTX. To obtain quasisteady-state $I-V$ relationships, we used slow voltage ramps (for details, see Materials and Methods). As shown in Figure 9A, repeated voltage ramps gave consistent results. Three ramps were averaged to construct $I-V$ curves.

An example of the $I-V$ curves obtained in the same B31/B32 under various conditions is shown in Figure $9 B$. Under control conditions, when the B31/B32 had been pharmacologically isolated with TTX, no inward current was seen (Fig. 9B, CNTRL). The addition of OXO caused a large shift in the $I-V$ relationship, producing a region of negative slope in the $I-V$ curve and a net inward current that peaked at approximately $-35 \mathrm{mV}$. Substitution of external sodium with NMDG eliminated the inward current (Fig. 9B, OXO $+0 \mathrm{Na}$ ), as did the addition of PIR (Fig. 9B, $\mathrm{OXO}+\mathrm{PIR})$.

To compare quantitatively across the different conditions and experiments, we compared the magnitude of the current at two voltages during the ramps, -65 and $-35 \mathrm{mV}$ (Fig. 9C). At -35 $\mathrm{mV}$, the current with OXO present was significantly different from the control, PIR, and sodium-free conditions (ANOVA: $F_{(7,42)}=19.3, p<0.001$; Bonferroni's multiple comparison test, $p<0.05)$ (Fig. 9C, asterisk), whereas at $-65 \mathrm{mV}$ there was no significant difference between the conditions. Thus, OXO induces at $-35 \mathrm{mV}$ a net inward current, carried primarily by so- dium and blocked by PIR, which is likely to underlie the plateau potential in the B31/B32 neurons.

\section{In the presence of $\mathrm{OXO}, \mathrm{B} 50$ no longer requires $\mathrm{B} 63$ firing to drive motor programs}

Because OXO could apparently replace the slow muscarinic synaptic input from B63 that is normally needed to induce the plateau potential in $\mathrm{B} 31 / \mathrm{B} 32$, we sought to examine whether $\mathrm{OXO}$ could replace the need for the input of B63 during B50-elicited motor programs. Extending the experimental paradigm already shown in Figure 1, we examined whether the ability of B50 to elicit motor programs was blocked by B63 hyperpolarization if OXO was present $(n=3)$ (Fig. 10). As before, B50 stimulation in normal saline (ASW; left traces) elicited a motor program containing a protraction phase with the B31/B32 plateau potential, followed by a retraction phase. If $\mathrm{B} 63$ was hyperpolarized at the onset of the B50 stimulation, none of this occurred (ASW; middle traces). With the addition of OXO, however, normal activity in response to the $\mathrm{B} 50$ stimulation was restored, although $\mathrm{B} 63$ continued to be hyperpolarized (OXO). Occasionally, immediately after the addition the OXO, OXO alone elicited two or three spontaneous cycles of activity without any B50 firing (Dembrow et al., 2003). After this, however, the preparation required B50 stimulation for motor programs to be elicited. In OXO, although the hyperpolarization of B63 caused some delay, B50 was nonetheless able to drive B31/B32 into plateau and drive the patterned nerve activity of protraction and retraction. In the presence of $\mathrm{OXO}$, the peak depolarization in $\mathrm{B} 31 / \mathrm{B} 32$ was $58.3 \pm 3.0 \mathrm{mV}$ without B63 hyperpolarization and $56.9 \pm 5.8 \mathrm{mV}$ with $\mathrm{B} 63$ hyperpolarization. These values are not significantly different from each other or from the peak depolarization in the absence of OXO, simply in normal saline (B31/B32 depolarization ANOVA as above; Bonferroni's multiple comparison tests, $p>0.05)$.

\section{Discussion}

In the architecture of CPG networks, neurons are typically connected via multiple pathways (Schmidt and Calabrese, 1992; Nusbaum and Beenhakker, 2002; Hurwitz et al., 2003). Information from one neuron may reach a follower neuron in several ways, converging onto the follower neuron through inputs that elicit apparently similar synaptic potentials. In this study, however, we have shown that the convergent synaptic inputs from two pathways by which B50 can excite B31/B32, directly and via B63, are not functionally equivalent. Although B50 and B63 each contain the same neurotransmitter and elicit similar synaptic potentials, the input from B63 is qualitatively different from the input from $\mathrm{B} 50$ in that $\mathrm{B} 63$ has privileged access to the plateau potential of B31/B32. Figure 11 shows the circuit schema, highlighting the singular significance of the input from B63.

\section{The B31/B32 plateau potential is conditional}

Induction of the B31/B32 plateau potential represents the committed step toward the initiation of a cycle of patterned motor activity. Despite its importance, the characterization of how the B31/B32 plateau potential is induced had been incomplete. Here, we have provided evidence that suggests that the plateau potential in B31/B32 is not an endogenous property of the neuron. Although directly injecting current into B31/B32 triggers the plateau potential (Susswein and Byrne, 1988; Hurwitz et al., 1994; Susswein et al., 2002), these current injections concomitantly transfer to B63 via electrical coupling (see Fig. 5), causing B63 to fire and activate its input to B31/B32. The B31/B32 plateau potential induction is conditional on this synaptic input. If the B63- 
B31/B32 feedback loop is broken, DC injections into B31/B32 no longer induce plateau potentials. Thus, blocking action potentials in B63 with TTX (Fig. 5) and/or HiDi solution (Fig. 6) prevents plateau potential induction. B31/B32 plateau potential induction in response to current injection can then be restored by the addition of the muscarinic agonist OXO (Figs. 5, 6). $\mathrm{OXO}$ activates the $\mathrm{B} 31 / \mathrm{B} 32$ plateau potential by introducing a negative-slope region in the steady-state $I-V$ relationship of B31/ B32 with a net inward current at depolarized potentials (Fig. 9). This inward current is blocked by the muscarinic antagonist PIR. Together, these findings strongly suggest that the B31/B32 plateau potential is a conditional property, activated in response to muscarinic input from B63.

Muscarinic agonists such as OXO have revealed conditional plateau potentials in neurons in many vertebrate and invertebrate systems (Gorczyca et al., 1991; Cattaert et al., 1994; Elson et al., 1994; Fraser and MacVicar, 1996; Kiehn et al., 1996; Svirskis and Hounsgaard, 1997). The OXO-induced current in B31/B32 appears to be mostly a sodium current (Figs. 8, 9). In both invertebrate and vertebrate systems, similar voltage-dependent, TTXinsensitive cationic currents have been suggested to contribute to plateau potentials in neurons believed to receive cholinergic inputs (Fraser and MacVicar, 1996; Rekling and Feldman, 1997; Kawasaki et al., 1999). Here, we show, however, that not all cholinergic inputs converging onto a neuron possessing a plateau potential conditional on muscarinic transmission are necessarily capable of activating the plateau potential. It is possible that, as in the case of B31/B32, other cholinergically activated plateau potentials may also be dependent on specific subsets of cholinergic inputs.

B31/B32 receives two classes of slow input, distinguishable by PIR sensitivity B31/B32 receives several putatively cholinergic synaptic inputs. The fast EPSPs elicited by each of these inputs are blocked by the nicotinic antagonist hexamethonium (Dembrow et al., 2003; Hurwitz et al., 2003). In addition to the fast EPSPs, each of these inputs also elicits a slow EPSP. The B63-elicited slow EPSP is blocked by the muscarinic antagonist PIR (Fig. 2). In contrast, the B50-elicited slow EPSP is PIR insensitive (Fig. 2). We have not yet determined the reason for the different pharmacological sensitivities of these two types of slow EPSPs in B31/B32. One possibility is that both slow EPSPs are cholinergic, with two different types of cholinergic receptors se-

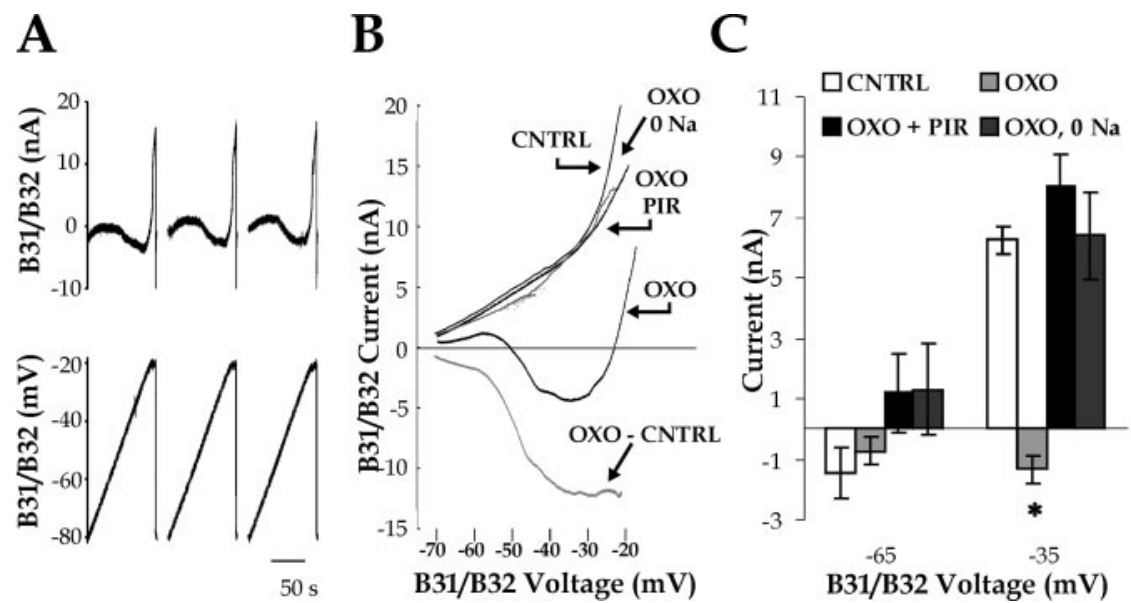

Figure 9. Oxotremorine (0X0) induces an inward current in voltage-clamped B31/B32. $A$, Example of the voltage ramps applied to B31/B32 ( V) and the resulting current ( $/$ ), in the presence of $0 \times 0$. Three consecutive voltage ramps, with a 20 sec delay between them, were applied from -80 to $-20 \mathrm{mV}$ at $0.5 \mathrm{mV} / \mathrm{sec}$ (see Materials and Methods). $B, I--V$ relationships for several experimental conditions obtained in the same B31/B32. The conditions were tested in the following order: (1) normal ASW with $3.3 \times 10^{-5} \mathrm{M} \mathrm{TTX}$ (CNTRL) (the TTX remained present in each of the subsequent conditions); (2) with the addition of $10^{-5} \mathrm{M} 0 \times 0$; (3) after substitution of external sodium with NMDG, in the presence of $10^{-5} \mathrm{M} 0 \mathrm{XO}(0 \mathrm{XO}+0 \mathrm{Na})$; (4) after reintroduction of external sodium in the presence of $10^{-5} \mathrm{M} O X 0$ (data not shown, because results were identical to the $0 \times 0$ condition); and (5) with the addition of $10^{-3} \mathrm{M}$ PIR to $10^{-5} \mathrm{M}$ OXO (OXO + PIR). In addition, the $I-V$ relationship of the net OXO-induced current (OXO - CNTRL) is plotted in gray. $C$, Group data. Currents measured from $/-V$ relationships like those in $B$ at $-65 \mathrm{mV}$ and $-35 \mathrm{mV}$ (CNTRL, $n=8 ; 0 \times 0, n=9 ; 0 \times 0$ $+0 \mathrm{Na}, n=3 ; 0 \times 0+\mathrm{PIR}, n=5)$. The asterisk indicates a statistically significant difference from CNTRL (see Results).

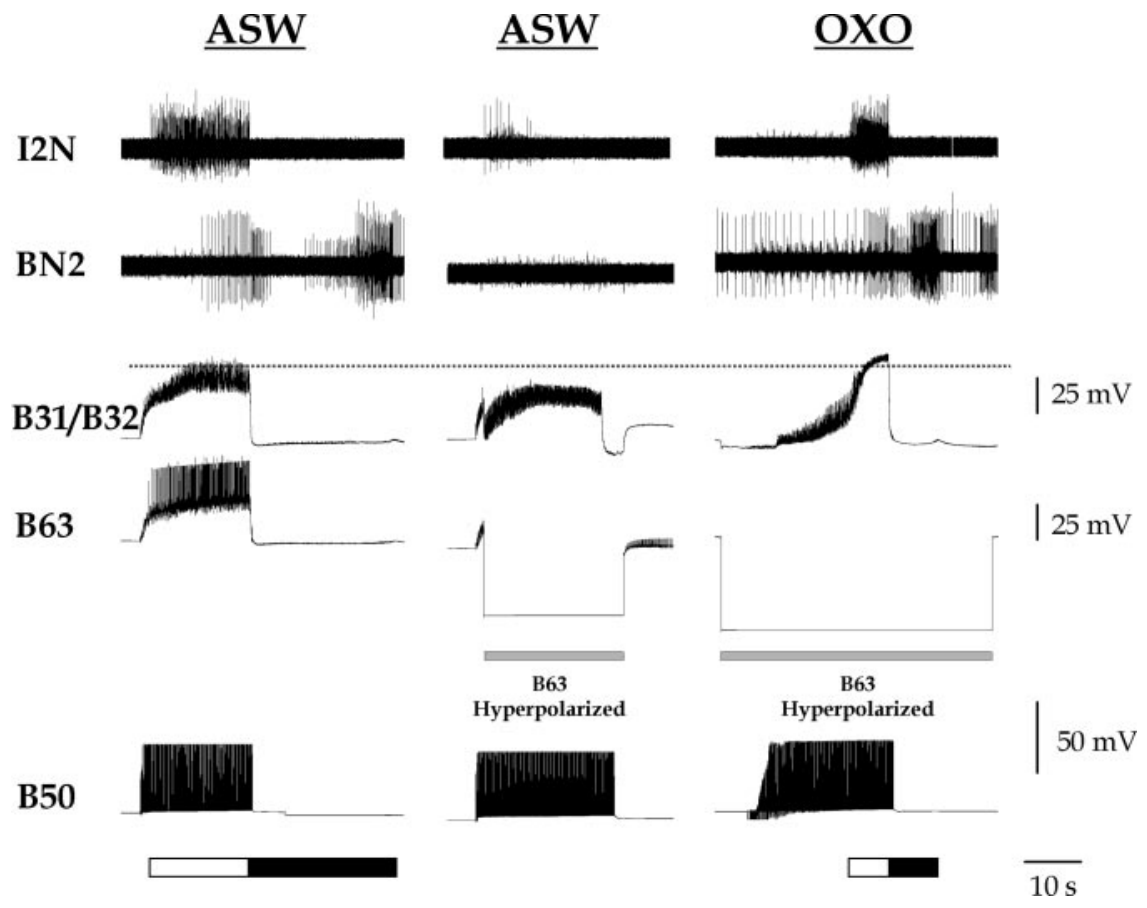

Figure 10. In the presence of $0 \times 0, B 50$ no longer requires $B 63$ firing to induce the plateau potential in $B 31 / B 32$ and motor programs. As in Figure $1 A$, single-cycle motor programs were elicited by stimulating $B 50$ with brief current pulses at $10 \mathrm{~Hz}$. The protraction phase (marked by white bar below traces) was monitored by firing of B31/B32 and activity in the I2 nerve (I2N). The retraction phase (marked by black bar below traces) was monitored by activity in the buccal nerve 2 (BN2). When B63 was hyperpolarized (gray bar) in normal saline (ASW; middle traces), the B31/B32 plateau potential was blocked and the I2N and BN2 activities were greatly diminished. $0 \times 0\left(10^{-5} \mathrm{M}\right)$ restored the $\mathrm{B} 31 / \mathrm{B} 32$ plateau potential and the $\mathrm{I} \mathrm{N}$ and $\mathrm{BN} 2$ activities even when B63 was hyperpolarized (right traces). The dashed line marks the level of peak depolarization in B31/B32 under control conditions to facilitate comparison.

lectively associated with them in B31/B32. Differential distribution of receptor types in the same neuron has been described (Rubio and Wenthold, 1997; Fritschy et al., 1998; Toth and McBain, 1998; Zhao et al., 1998). 


\section{Core Protraction Circuitry}

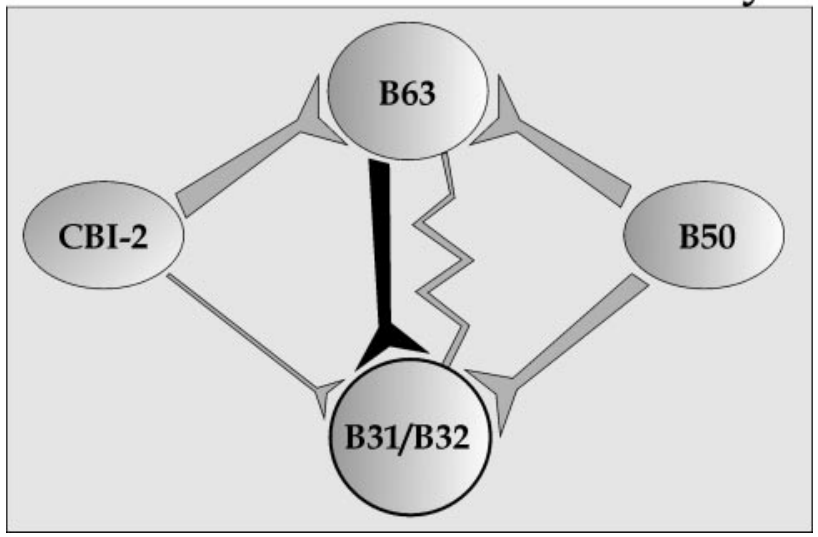

Figure 11. Circuit schema resulting from this work. Although multiple excitatory inputs converge onto neuron B31/B32 in the protraction-phase feeding circuitry of Aplysia, these inputs are not equivalent. Only the input from B63 (black) elicits a PIR-sensitive slow EPSP in B31/B32 and is critical for induction of the plateau potential and motor programs. Other inputs elicit PIR-insensitive slow EPSPs (gray). The input from (BI-2 is weak (Hurwitz et al., 2003), and its slow EPSP has not been tested. The zig-zagged line represents electrical coupling between $B 63$ and $B 31 / B 32$.

Alternatively, the B50-elicited slow EPSP may not be cholinergic. In addition to ACh, B50 contains a neuropeptide cotransmitter, PRQFVa (Dembrow et al., 2003). In principle, PRQFVa might mediate the PIR-insensitive EPSP. However, bath application of PRQFVa does not change the resting membrane potential of B31/B32 (our unpublished observations). In any case, even if the B50-elicited slow EPSP is not cholinergic, it still appears that both $\mathrm{B} 50$ and $\mathrm{B} 63$ release ACh onto B31/B32, as evidenced by the fast hexamethonium-sensitive EPSPs, yet only the B63 input elicits the PIR-sensitive slow EPSP. This again points to differential distribution of receptors. Differential receptor distribution may thus be the means by which one input has preferential access to the plateau potential in $\mathrm{B} 31 / \mathrm{B} 32$.

\section{B31/B32 plateau potential activation in the Aplysia feeding CPG}

Our data suggest that the B31/B32 plateau potential is specifically conditional on synaptic input from B63. Of course, we cannot completely rule out the possibility that other, yet uncharacterized, neurons may also contribute to the induction of the plateau potential. However, the importance of $\mathrm{B} 63$ for plateau induction is evident in that when even one B63 is hyperpolarized during motor programs triggered by either B50 (Fig. 1) or CBI-2 (Hurwitz et al., 1997, 2003) stimulation, the B31/B32 plateau potential is no longer induced, and indeed the entire motor program is prevented.

There are three identified components of the B63 to B31/B32 synaptic connection: the fast hexamethonium-sensitive EPSPs, the slow PIR-sensitive EPSPs, and electrotonic EPSPs. Of these, it is the slow PIR-sensitive component that appears critical for plateau potential induction. PIR at $1 \mathrm{~mm}$, which blocks the B63elicited slow EPSP in B31/B32 (Fig. 2), also blocks the B31/B32 plateau potential whether it is induced by B50 stimulation (Fig. 3), CBI-2 stimulation (Hurwitz, DiCaprio, and Weiss, unpublished observations), or DC injection into B31/B32 (Fig. 4). In contrast, blocking the fast EPSPs delays but does not block the B31/B32 plateau potential (Hurwitz et al., 2003). With synaptic input to B31/B32 eliminated, OXO restores the induction of the plateau potential, and this again is blocked by $1 \mathrm{mM}$ PIR. Finally,
OXO removes the requirement for $\mathrm{B} 63$ firing in the induction of the B31/B32 plateau potential and motor programs (Fig. 10).

To induce the plateau potential in B31/B32, two events appear to be necessary: (1) the activation of the slow PIR-sensitive current by the input from B63 and (2) the depolarization of B31/B32 such that this current can trigger the regenerative plateau potential. We hypothesize that the following events underlie B31/B32 plateau potential induction during a motor program. B50 and CBI-2 excite B31/B32 and B63. B63 begins to spike, and B31/B32 begins to be depolarized by the summated fast EPSPs from B63, B50, and CBI-2. Some of this depolarization in B31/B32 transfers electronically to B63, increasing its firing frequency. This positive feedback loop continues until the slow, PIR-sensitive synaptic transmission from B63 is of sufficient magnitude to induce the plateau potential in $\mathrm{B} 31 / \mathrm{B} 32$. As the slow synaptic current from B63 is magnified at more depolarized membrane potentials (Fig. 2 ), the direct depolarizing input from B50 or CBI-2 can determine the latency with which the B31/B32 plateau potential is induced. B50 induces the plateau potential in B31/B32 much sooner than does CBI-2 (Dembrow et al., 2003). This difference in the timing of the induction is likely to be attributable to the differences in the strength of the direct synaptic connections of B50 and CBI-2 to B31/B32 (Dembrow et al., 2003; Hurwitz et al., 2003).

\section{Implications for convergence in network function}

Convergence is a ubiquitous motif in the architecture of neuronal networks. Neurons that receive converging inputs integrate them and express this integration through their activity. Often, this integration is considered to be the simple summation of the magnitudes of various inhibitory and excitatory inputs. As we have shown here, however, an additional dimension may need to be considered when examining converging inputs. Our study suggests that rather than simply possessing different magnitudes, certain inputs may also be qualitatively distinct in that they may have privileged access to critical properties of their targets. Our findings reinforce the conclusion that the description of all of the monosynaptic connections within a neuronal network is insufficient to understand how the network operates (Selverston, 1980; Getting, 1989; Marder and Calabrese, 1996). The fact that converging inputs are not necessarily all functionally equivalent has implications for current conceptualizations of how neuronal networks process information.

\section{References}

Abbott L, Sejnowski T (1999) Neural codes and distributed representations, foundations of neural computation. Cambridge, MA: MIT.

Amit DJ (1989) Modeling brain function, the wold of attractor neural networks. New York: Cambridge UP.

Cattaert D, Araque A, Buno W, Clarac F (1994) Nicotinic and muscarinic activation of motoneurons in the crayfish locomotor network. J Neurophysiol 72:1622-1633.

Dembrow NC, Jing J, Brezina V, Weiss KR (2002) A specific synaptic pathway activates the plateau potential responsible for the maintenance of the protraction phase in the Aplysia feeding central pattern generator. Soc Neurosci Abstr 28:367.13.

Dembrow NC, Jing J, Proekt A, Romero A, Vilim FS, Cropper EC, Weiss KR (2003) A newly identified buccal interneuron initiates and modulates feeding motor programs in Aplysia. J Neurophysiol 90:2190-2204.

Elson RC, Panchin YV, Arshavsky YI, Selverston AI (1994) Multiple effects of an identified proprioceptor upon gastric pattern generation in spiny lobsters. J Comp Physiol [A] 174:317-329.

Fraser DD, MacVicar BA (1996) Cholinergic-dependent plateau potential in hippocampal CA1 pyramidal neurons. J Neurosci 16:4113-4128.

Fritschy JM, Johnson DK, Mohler H, Rudolph U (1998) Independent assembly and subcellular targeting of GABA(A)-receptor subtypes demon- 
strated in mouse hippocampal and olfactory neurons in vivo. Neurosci Lett 249:99-102.

Getting P (1989) Emerging principles from central pattern generator networks. Annu Rev Neurosci 12:185-204.

Gorczyca MG, Budnik V, White K, Wu CF (1991) Dual muscarinic and nicotinic action on a motor program in Drosophila. J Neurobiol 22:391-404.

Hurwitz I, Goldstein RS, Susswein AJ (1994) Compartmentalization of pattern-initiation and motor functions in the $\mathrm{B} 31$ and $\mathrm{B} 32$ neurons of the buccal ganglia of Aplysia californica. J Neurophysiol 71:1514-1527.

Hurwitz I, Neustadter D, Morton DW, Chiel HJ, Susswein AJ (1996) Activity patterns of the B31/B32 pattern initiators innervating the $\mathrm{I} 2$ muscle of the buccal mass during normal feeding movements in Aplysia californica. J Neurophysiol 75:1309-1326.

Hurwitz I, Kupfermann I, Susswein AJ (1997) Different roles of neurons B63 and B34 that are active during the protraction phase of buccal motor programs in Aplysia californica. J Neurophysiol 78:1305-1319.

Hurwitz I, Kupfermann I, Weiss KR (2003) Fast synaptic connections from CBIs to pattern-generating neurons in Aplysia: initiation and modification of motor programs. J Neurophysiol 89:2120-2136.

Kawasaki H, Palmieri C, Avoli M (1999) Muscarinic receptor activation induces depolarizing plateau potentials in bursting neurons of the rat subiculum. J Neurophysiol 82:2590-2601.

Kiehn O, Johnson BR, Raastad M (1996) Plateau properties in mammalian spinal interneurons during transmitter-induced locomotor activity. Neuroscience 75:263-273.

Kristan Jr WB, Shaw BK (1997) Population coding and behavioral choice. Curr Opin Neurobiol 7:826-831.

Kupfermann I (1974) Feeding behavior in Aplysia: a simple system for the study of motivation. Behav Biol 10:1-26.

Marder E, Calabrese RL (1996) Principles of rhythmic motor pattern generation. Physiol Rev 76:687-717.
Morton DW, Chiel HJ (1993) In vivo buccal nerve activity that distinguishes ingestion from rejection can be used to predict behavioral transitions in Aplysia. J Comp Physiol [A] 172:17-32.

Nusbaum MP, Beenhakker MP (2002) A small-systems approach to motor pattern generation. Nature 417:343-350.

Rekling JC, Feldman JL (1997) Calcium-dependent plateau potentials in rostral ambiguus neurons in the newborn mouse brain stem in vitro. J Neurophysiol 78:2483-2492.

Rubio ME, Wenthold RJ (1997) Glutamate receptors are selectively targeted to postsynaptic sites in neurons. Neuron 18:939-950.

Schmidt J, Calabrese RL (1992) Evidence that acetylcholine is an inhibitory transmitter of heart interneurons in the leech. J Exp Biol 171:329-347.

Selverston AI (1980) Are central pattern generators (CPGs) understandable? Brain Behav Sci 3:535-571.

Susswein AJ, Byrne JH (1988) Identification and characterization of neurons initiating patterned neural activity in the buccal ganglia of Aplysia. J Neurosci 8:2049-2061.

Susswein AJ, Hurwitz I, Thorne R, Byrne JH, Baxter DA (2002) Mechanisms underlying fictive feeding in Aplysia: coupling between a large neuron with plateau potentials activity and a spiking neuron. J Neurophysiol 87:2307-2323.

Svirskis G, Hounsgaard J (1997) Depolarization-induced facilitation of a plateau-generating current in ventral horn neurons in the turtle spinal cord. J Neurophysiol 78:1740-1742.

Toth K, McBain CJ (1998) Afferent-specific innervation of two distinct AMPA receptor subtypes on single hippocampal interneurons. Nat Neurosci 1:572-578.

Zhao HM, Wenthold RJ, Petralia RS (1998) Glutamate receptor targeting to synaptic populations on Purkinje cells is developmentally regulated. J Neurosci 18:5517-5528. 